\section{Dengue, HIV and Thrombocytopenia}

Sir,

We read with great interest the reports on two patients with thrombocytopenia by Kohli et al and Verma et al published recently in the journal. ${ }^{1,2}$ We wish to make some observations in relation to these two reports.

In the letter by Verma et al it would have been useful to know the lymphocyte count in the child when he first presented with epistaxis. ${ }^{2}$ Although, the total leucocyte count was mentioned, the differential count was not included. We often tend to focus our attention only on the neutrophil count, and tend to neglect the significance of a low age corrected lymphocyte count. The observation of a low lymphocyte count for age in this child at his first presentation may have helped the clinical team to consider further investigations at an earlier stage.

Secondly, the initial presentation of immune thrombocytopenic purpura (ITP) could be secondary to other immune-mediated diseases, such as tuberculosis which can easily go undetected due its subtle presentation in children. An increasing number of cases of ITP secondary to tuberculosis has been reported in children. ${ }^{3}$

Thirdly, taking a detailed family history should be given special attention. We have come across cases from rural regions of North India where mothers or other relatives have died from tuberculosis, with the children or other parent later found to have manifestations of HIV.

In the report by Kohli et al, it would be useful to know the child's past medical history particularly if the child had problems with bleeding before he developed dengue. ${ }^{1}$ Dengue infections could worsen thrombocytopenia in a pre-existent condition like ITP. It would have been more instructive if the doses of steroids administered to this child (presumably for ARDS) were included in the report. This would have given us a better idea on how steroid refractory the presumed immune thrombocytopenia was. It was also interesting that despite the child having DSS, his liver function tests remained normal.

The possibility of co-existing infections such as scrub typhus and malaria should be explored rigorously in children with persistent thrombocytopenia and dengue infections. ${ }^{4}$ The report failed to mention if these were looked for and excluded.

The authors have rightly pointed that the evidence base for IVIG use in dengue is limited. Interestingly a recent randomised controlled trial studied this aspect in a small number of patients with secondary dengue. ${ }^{5}$ The authors found that high dose IVIG had no effect in hastening the recovery of platelet counts patients with secondary dengue infections, and thus concluded that platelet clearance by macrophages through Fc gamma receptors is not a primary mechanism in pathogenesis of thrombocytopenia in dengue infections.

Pankaj Garg and ${ }^{1}$ Suranjith L. Seneviratne Department of Pediatrics and Clinical Epidemiology, Sitaram Bhartia Institute of Science and Research, New Delhi, India, 'St Mary's Hospital and Imperial College, London, UK E-mail: Suranjith.Seneviratne@imperial.nhs.uk [DOI - 10.1007 / S12098-008-0175-6]

\section{REFERENCES}

1. Kohli U, Saharan S, Lodha R, Kabra SK. Persistent thrombocytopenia following dengue shock syndrome. Indian J Pediatr 2008; 75 : 82-83.

2. Verma S, Gupta P. HIV infection masquerading as idiopathic thrombocytopenic purpura. Indian J Pediatr 2008; 75 : 85.

3. Krishnamurthy S, Yadav S. Immune thrombocytopenic purpura as a presentation of childhood tuberculosis. Indian J Pediatr 2007; 74: 853-855.

4. Pancharoen C, Thisyakorn U. Coinfections in dengue patients. Pediatr Infect Dis J 1998; 17 : 81-82.

5. Dimaano EM, Saito M, Honda S, Miranda EA, Alonzo MT, Valerio MD et al. Lack of efficacy of high-dose intravenous immunoglobulin treatment of severe thrombocytopenia in patients with secondary dengue virus infection. Am J Trop Med Hyg 2007; 77 : 1135-1138. 